\title{
(6) OPEN ACCESS \\ An outcomes evaluation of an emergency department early pregnancy assessment service and early pregnancy assessment protocol
}

For numbered affiliations see end of article.

\section{Correspondence to} Kim Wendt, Emergency Department, Capricorn Coast Hospital and Health Services, Hoskyn Drive, Yeppoon, QLD, 4073, Australia;

kim_wendt@health.qld.gov.au

Received 26 May 2013 Revised 2 September 2013 Accepted 4 September 2013 Published Online First 17 October 2013

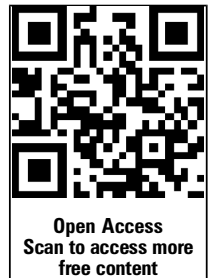

CrossMark

To cite: Wendt K, Crilly J, May $C$, et al. Emerg Med J 2014;31:e50-e54.

\author{
Kim Wendt, ${ }^{1,2,3}$ Julia Crilly, ${ }^{4,5,6}$ Chris May, ${ }^{1,7}$ Kym Bates, ${ }^{1}$ Rakhee Saxena ${ }^{1}$
}

\begin{abstract}
Background Complications in early pregnancy, such as threatened or actual miscarriage is a common occurrence resulting in many women presenting to the emergency department (ED). Early pregnancy service delivery models described in the literature vary in terms of approach, setting and outcomes. Our objective was to determine outcomes of women who presented to an Australian regional ED with diagnoses consistent with early pregnancy complications following the implementation of an early pregnancy assessment service (EPAS) and early pregnancy assessment protocol (EPAP) in July 2011.

Methods A descriptive, comparative (6 months before and after) study was undertaken. Data were extracted from the hospital ED information system and medical healthcare records. Outcome measures included: time to see a clinician, ED length of stay, admission rate, re-presentation rate, hospital admission and types of pathology tests ordered.
\end{abstract}

Results Over the 12 -month period, 584 ED presentations were made to the ED with complications of early pregnancy (268 PRE and 316 POST EPASEPAP). Outcomes that improved statistically and clinically following implementation included: time to see a clinician (decreased by 6 min from 35 to $29 \mathrm{~min}$ ), admission rate (decreased $6 \%$ from $14.5 \%$ to $8.5 \%$ ), increase in $\beta$-human chorionic gonadotrophin ordering by $10 \%$ (up to $80 \%$ POST), increase in ultrasound (USS) performed by $10 \%$ (up to $73 \%$ POST) and increase in pain score documentation by $23 \%$ (up to $36 \%$ POST). Conclusions The results indicate that patient and service delivery improvements can be achieved following the implementation of targeted service delivery models such as EPAS and EPAP in the ED.

\section{INTRODUCTION}

With more than one in five pregnancies ending in miscarriage, ${ }^{12}$ a large number of women present to the emergency department (ED) with associated complications such as pain and/or bleeding. ${ }^{3} 4$ Feelings such as inadequacy, confusion, anger, blaming and varying levels of grief can occur with pregnancy loss. As such, the provision of emotional care as well as clinical treatment is required if these women present to the ED. This requires sensitivity and preferably a calm and private environment to best achieve positive, lasting effects. ${ }^{25}$

Improvements in diagnostic and therapeutic interventions over time have resulted in changes to intervention approaches and treatment options for women who have complications of early pregnancy. Approaches described from the UK, Canada, New Zealand and more recently, Australia involve the use of specific protocols, care management guidelines and/or specific clinics or services. These approaches can result in positive patient and health service outcomes such as cost-effective care, improved quality of care, timely care and a reduced need for hospital admission. ${ }^{2}{ }^{4}$ 6-8 The basic premise of an ED-based early pregnancy assessment clinic/early pregnancy assessment service (EPAC/ EPAS) is to improve timeliness of care delivery and to provide continuity of care to women presenting to the ED who are $<20$ weeks gestation and have pain and/or vaginal bleeding. ${ }^{8} 9$

An EPAS coupled with an early pregnancy assessment protocol (EPAP) was implemented in a regional Australian ED in July 2011. The EPAS was a primarily nurse practitioner (NP)-led model with multidisciplinary input (ie, from ED doctors, ED nurses, social worker, obstetrics and gynaecology $(\mathrm{O} \& \mathrm{G})$ doctor and radiology) as required during business hours and medically led using a protocoldriven approach during out-of-business hours. The goal of the EPAS-EPAP model established within the ED was to primarily reduce ED waiting time to see a clinician, total ED length of stay (LOS), admission rate, nursing observations performed and pain score documentation. The aim of this study was to describe and compare outcomes of all women who presented to the ED with complications of early pregnancy for the 6 months pre-EPAS-EPAP implementation and post-EPAS-EPAP implementation.

\section{METHODS}

\section{Study design and setting}

A descriptive, comparative (before and after) design was used to describe and compare patient and service delivery outcomes (time to see a clinician, ED LOS, ED re-presentation rate, hospital admission rate and types of pathology tests ordered) at 6 months before (1 January-30 June 2011-PRE) and after (1 July-31 December 2011-POST) the implementation of the EPAS-EPAP.

The region surrounding the study hospital had approximately 138670 residents of Redland City, including surrounding island communities and 30000 people in the Wynnum Manly area of Brisbane's Bayside. ${ }^{10} 11$ The study site was a public, teaching hospital with 138 beds (31 in the ED). In 2009/2010, there were 44284 (paediatric and adult) $\mathrm{ED}$ attendances, with an admission rate of $15 \% .{ }^{11}$

\section{Sample}

Inclusion criteria for this study were ED presentations made during 1 January 2011-31 December 2011, women, aged 16-49 years, with emergency 
department information system (EDIS) International Classification of Diseases (ICD-10AM) codes: O02.1 (missed miscarriage/abortion), O03.9 (inevitable/complete miscarriage), O20.0 (threatened miscarriage) and O00.9 (ectopic pregnancy). The ICD is the international standard diagnostic classification tool for all general epidemiological, health management and clinical purposes. ${ }^{12}$ Exclusion criteria were women who were not pregnant, heavy per vaginal (PV) bleeding (ie, more than normal period blood loss), ${ }^{13}$ haemodynamically unstable, hyperemesis, requiring opioids for pain management, $>20$ weeks gestation, complicating medical conditions, known ectopic pregnancy and those requiring $O \& G$ specialist follow-up post-procedure.

\section{Data collection}

Data were collected from electronic and paper-based medical healthcare records. Table 1 presents data obtained from each information source. Clinical data collected were based on previous research examining early pregnancy service outcomes. ${ }^{7}$ The Australasian Triage Scale is an indicator of urgency which ensures patients are seen in order of priority-Category 1immediately, Category 2-within $10 \mathrm{~min}$, Category 3-within $30 \mathrm{~min}$, Category 4-within $60 \mathrm{~min}$ and Category 5-within 120 min. ${ }^{14}$ Pathology data collected were based on recommended investigations (full blood count (FBC), quantitative $\beta$-human chorionic gonadotrophin (BHCG) and blood group/ Rhesus factor status) that should be performed on women who present with complications of early pregnancy. ${ }^{2}$ EDIS data are routinely collected in a prospective fashion by ED clinical staff and were provided upon request to the researchers from the hospital decision support services personnel in Excel (Microsoft Corp., Redmond, Washington, USA) format.

\section{Outcomes measured}

Outcomes measured included time to see a clinician (primary outcome), ED LOS, admission rate and unplanned ED re-presentation rates ( $<24 \mathrm{~h}$ and $>48 \mathrm{~h}$ ) (secondary outcomes). These were defined as follows:

- Time to see a clinician: time ( $\mathrm{min}$ ) from triage presentation to treating clinician (medical officer (MO) or NP) contact.

- ED LOS: time (min) from triage presentation to ED discharge/hospital admission.

- Admission: any patient who is recorded on the EDIS as 'admitted'.

- Unplanned re-presentations: any patient who re-presented to the same ED within $24 \mathrm{~h}$ or after $48 \mathrm{~h}$ of initial presentation,

Table 1 Data obtained from information sources

\begin{tabular}{|c|c|}
\hline Data source & Data provided/collected \\
\hline EDIS & $\begin{array}{l}\text { Patient age; gestation; ATS category; reason for presentation; } \\
\text { date and time of arrival; day of week; triage allocation area } \\
\text { (acute/non-acute); date and time seen by doctor or clinician; } \\
\text { clinician type (doctor/nurse practitioner); total ED length of } \\
\text { stay; obstetrics and gynaecology registrar involvement; } \\
\text { discharge diagnosis (ICD-10 code); discharge destination from } \\
\text { ED (admitted/discharged); re-presentation to ED }\end{array}$ \\
\hline $\begin{array}{l}\text { Healthcare } \\
\text { records }\end{array}$ & $\begin{array}{l}\text { Observations performed at triage }(\mathrm{Y} / \mathrm{N}) \text {; pain score recorded } \\
(\mathrm{Y} / \mathrm{N}) \text {; type of pathology and medical imaging investigations } \\
\text { performed; treatment or management plan provided } \\
\text { (expectant, medical or surgical intervention) }\end{array}$ \\
\hline
\end{tabular}

ATS, Australasian Triage Scale; ED, emergency department; EDIS, emergency department information system; ICD, International Classification of Diseases; $N$, no; $Y$, yes. not for planned follow-up or prearranged treatment (such as surgical management).

\section{Data analysis}

The demographic (age), ED characteristics (eg, reason for presentation, triage category, shift of presentation) and outcomes of the sample were described using descriptive statistics. Specifically, median and IQR were used for continuous (age and time) data and frequencies and percentages were used for grouped data (eg, triage category, reason for presentation). Pre-EPAS-EPAP implementation and post-EPAS-EPAP implementation differences were identified using inferential statistics; specifically, Mann-Whitney U test for non-parametric continuous outcome data (eg, time to see a clinician, ED LOS) and $\chi^{2}$ test for categorical data (eg, unplanned re-presentation to the ED, hospital admission, number/type of pathology and other tests ordered). All data were analysed using SPSS V.18.0 (SPSS, Illinois, USA). A p value of $<0.05$ was considered statistically significant.

Approvals to conduct this study were attained from the Health Service District and University human research ethics committee and Queensland Health's research ethics and governance unit.

\section{RESULTS}

\section{Patient demographics}

A total of 49830 presentations were made to the study site ED during the study period (24 881pre-implementation and 24949 post-implementation). Of these, 584 women (268 PRE; 316 POST EPAS-EPAP implementation) aged 16-49 years with a diagnosis consistent with complications of early pregnancy presented to the ED during the 12 -month study period. The median age for all women was 30 years (IQR: 25-34, range: 16-45). Age did not differ significantly between PRE and POST groups $(\mathrm{p}=0.30)$.

\section{ED characteristics}

ED characteristics (diagnosis, triage category and shift of arrival) of women presenting to the ED with early pregnancy-related complications did not differ significantly between PRE and POST groups (see table 2).

\section{ED outcomes}

ED outcomes measured in this study (time to see a clinician, ED LOS, O\&G referral/consult, hospital admission, unplanned re-presentations within $24 \mathrm{~h}$ and greater than $48 \mathrm{~h}$ ) are presented in table 3 . The overall median time waiting to be seen by a clinician was $31 \mathrm{~min}$ (IQR: $15-60 \mathrm{~min}$ ). This differed significantly PRE versus POST EPAS-EPAP (35 vs $29 \mathrm{~min}, \mathrm{p}=0.03$ ). The overall median ED LOS was 165 min (IQR 96-250 min). A statistically significant difference between PRE and POST EPASEPAP ED LOS time frames was not identified (173 vs $152 \mathrm{~min}$, $\mathrm{p}=0.09$ ). Approximately one-third of ED presentations over the 12 -month period were referred to O\&G. This did not change significantly PRE versus POST EPAS-EPAP implementation. Most women were discharged home from the ED, with around $10 \%$ requiring hospital admission. Admission rate decreased statistically and clinically (by 6\% PRE vs POST EPAS-EPAP).

Subgroup analyses were performed to identify time to see a clinician (NP or MO) for the PRE and POST groups. For the PRE group, NPs saw 82 (30.6\%) of the 268 patient presentations; MOs saw the other $69.4 \%$. For the POST group, NPs saw $125(39.6 \%)$ of the patient presentations; MOs saw the other $64.6 \%$. While time to see a clinician decreased in both clinician 
Table 2 ED characteristics for female presentations with complications associated with early pregnancy

\begin{tabular}{lccc}
\hline & $\begin{array}{l}\text { Pre-EPAS- } \\
\text { EPAP } \\
\text { n=268 (\%) }\end{array}$ & $\begin{array}{l}\text { Post-EPAS- } \\
\text { EPAP } \\
\mathbf{n}=316(\%)\end{array}$ & $\begin{array}{l}\mathbf{p} \\
\text { Value }\end{array}$ \\
\hline ED characteristics & $29(25-34)$ & $30(24-34)$ & 0.30 \\
\hline Median (IQR) age & & & 0.53 \\
Diagnosis & $130(48.5)$ & $151(47.8)$ & \\
ICD 020.0 (threatened miscarriage) & $110(41.0)$ & $133(42.1)$ & \\
ICD 003.9 (inevitable/complete & & & \\
miscarriage) & $15(5.6)$ & $23(7.3)$ & \\
ICD 002.1 (missed miscarriage) & $13(4.9)$ & $9(2.8)$ & \\
ICD 000.9 (ectopic pregnancy) & & & \\
Triage category & $3(1.1)$ & $5(1.6)$ & \\
ATS 2 & $115(42.9)$ & $132(41.8)$ & \\
ATS 3 & $119(44.4)$ & $120(38.0)$ & \\
ATS 4 & $31(11.6)$ & $59(18.7)$ & \\
ATS 5 & & & \\
Shift arrived & $146(54.5)$ & $197(62.3)$ & \\
Day (07:00-14:59) & $103(38.4)$ & $102(32.3)$ \\
Evening (15:00-22:59) & $19(7.1)$ & $17(5.4)$ & \\
Night (23:00-06:59) & & & \\
\hline ATS, Australasian Triage Scale; ED, emergency department; EPAP, early pregnancy \\
assessment protocol; EPAS, early pregnancy assessment service; ICD, International \\
Classification of Diseases.
\end{tabular}

treating groups, this outcome was only significant in the NP group. Subgroup analyses were also performed to identify whether ED LOS differed by treating clinician (NP or MO) between PRE and POST. While ED LOS decreased in both clinician treating groups, this was not statistically significant.

Table 3 ED outcomes for female patient presentations with complications associated with early pregnancy

\begin{tabular}{|c|c|c|c|}
\hline ED outcomes & $\begin{array}{l}\text { Pre-EPAS-EPAP } \\
n=268(\%)\end{array}$ & $\begin{array}{l}\text { Post-EPAS-EPAP } \\
n=316(\%)\end{array}$ & $\begin{array}{l}p \\
\text { Value }\end{array}$ \\
\hline $\begin{array}{l}\text { Median (IQR) time to see a } \\
\text { clinician }\end{array}$ & $35(16-74)$ & $29(14-54)$ & 0.03 \\
\hline Medical officer & $37(16-75)$ & $36(19-63)$ & 0.78 \\
\hline Nurse practitioner & $30(14-70)$ & $22(11-41)$ & 0.006 \\
\hline Median (IQR) ED LOS & $173(107-260)$ & $152(89-240)$ & 0.09 \\
\hline Medical officer & $189(112-275)$ & $188(104-265)$ & 0.36 \\
\hline Nurse practitioner & $145(80-193)$ & $121(71-199)$ & 0.09 \\
\hline OG Referral/consultation & & & 0.52 \\
\hline No & $173(64.6)$ & $212(67.1)$ & \\
\hline Yes & $95(35.4)$ & $104(32.9)$ & \\
\hline Admission status & & & 0.02 \\
\hline Home & $226(85.4)$ & $289(91.5)$ & \\
\hline Hospital admission* & $39(14.6)$ & $27(8.5)$ & \\
\hline Number of women & $\mathrm{N}=191(\%)$ & $\mathrm{N}=188(\%)$ & \\
\hline $\begin{array}{l}\text { Women with one only } \\
\text { presentation }\end{array}$ & $128(67.0)$ & $114(60.6)$ & \\
\hline $\begin{array}{l}\text { Women with }>1 \\
\text { presentation }\end{array}$ & $63(33.0)$ & $74(39.4)$ & \\
\hline \multicolumn{4}{|l|}{ ED re-presentation } \\
\hline $\begin{array}{l}\text { Re-presentation to ED } \\
<24 \text { h (yes) }\end{array}$ & $47 / 77(61.0)$ & $53 / 128$ (41.4) & \\
\hline $\begin{array}{l}\text { Re-presentation to ED } \\
>48 \text { h (yes) }\end{array}$ & $30 / 77(39.0)$ & $75 / 128(58.6)$ & \\
\hline
\end{tabular}

A total of 379 women (191 PRE and 188 POST EPAS-EPAP) made the 584 ED presentations. Most women (64\%) presented to the ED on one occasion only; however, there were some who re-presented on more than one occasion. A higher number of ED re-presentations were noted in the post-EPAS-EPAP time frame compared with the pre-EPAS-EPAP time frame. Most of these occurred $>48 \mathrm{~h}$ after leaving the ED. This is likely reflective of the decreased admission rate.

\section{Clinical investigations}

Clinical investigations (that include pathology, pelvic ultrasound and pelvic exam) were identified from reviewing healthcare records of 581 of the 584 presentations (267 PRE and 314 POST EPAS-EPAP). These are presented in table 4. For three cases, the healthcare records were unavailable. Pathology was ordered on $75.9 \%(n=441)$ of the 581 female presentations. Ordering rates did not differ significantly PRE versus POST EPAS-EPAP implementation (PRE: $\mathrm{n}=197,73.8 \%$ vs POST $\mathrm{n}=244,77.7 \%, \mathrm{p}=0.27$ )

Regarding the type of pathology test ordered, the majority of all women received a FBC (65\%) and BHCG $(75 \%)$ when they presented to the ED as part of their primary initial investigation. The proportion of BHCG tests increased by $10 \%$ (to $80 \%$ ) following EPAS-EPAP implementation. This was significant. Other miscellaneous tests decreased from PRE to POST and the proportion of ultrasounds (USS) performed increased (by 10\%) to $73 \%$. These were significant (see table 4 ).

\section{Specific point-of-care events}

The healthcare records of the women were also reviewed in order to provide further in-depth details regarding specific point-of-care events (see table 5). The median gestation for all women was 8 weeks (IQR 6-10). This did not differ between PRE and POST groups. Most women (75\%) had observations performed at triage. This did not differ when PRE and POST groups were compared. A quarter of all women had a pain scale documented. This increased (significantly) from PRE to POST (13\% vs 36\%, p<0.001). Most women had an expectant medical plan. While most women were provided with some form of follow-up, the proportion of women receiving no follow-up decreased from PRE to POST EPAS-EPAP implementation.

\section{DISCUSSION}

\section{Growth}

The resident population of Redland City-the main catchment of Redland Hospital-is forecast to increase to $37.6 \%$ (from

Table 4 Investigations for female presentations with complications associated with early pregnancy

\begin{tabular}{lcccc}
\hline & $\begin{array}{l}\text { Pre-EPAS- } \\
\text { EPAP } \\
\mathbf{n}=\mathbf{2 6 7}(\%)\end{array}$ & $\begin{array}{l}\text { Post-EPAS- } \\
\text { EPAP } \\
\mathbf{n}=314(\%)\end{array}$ & $\begin{array}{l}\text { Total } \\
\mathbf{n}=581 \\
(\%)\end{array}$ & $\begin{array}{l}\mathbf{p} \\
\text { Value }\end{array}$ \\
\hline BHCG & $188(70.4)$ & $251(79.9)$ & $439(75.6)$ & 0.008 \\
FBC & $177(66.3)$ & $198(63.1)$ & $375(64.5)$ & 0.417 \\
Blood group & $142(53.2)$ & $160(51.0)$ & $302(52.0)$ & 0.592 \\
Cross match & $15(5.6)$ & $13(4.1)$ & $28(4.8)$ & 0.407 \\
Other (miscellaneous) & $63(23.6)$ & $49(15.6)$ & $112(19.3)$ & 0.015 \\
Pelvic ultrasound & $169(63.3)$ & $229(72.9)$ & $398(68.5)$ & 0.013 \\
Pelvic exam & $74(27.7)$ & $92(29.3)$ & $166(28.6)$ & 0.674
\end{tabular}

Data presented as $n(\%) ; p$ value based on $\chi^{2}$ test.

BHCG, $\beta$-human chorionic gonadotrophin; EPAP, early pregnancy assessment protocol; EPAS, early pregnancy assessment service; $F B C$, full blood count. 
Table 5 Specific point-of-care events for female patients presenting with complications associated with early pregnancy

\begin{tabular}{|c|c|c|c|}
\hline Care event & $\begin{array}{l}\text { Pre-EPAS- } \\
\text { EPAP } \\
n=267(\%)\end{array}$ & $\begin{array}{l}\text { Post-EPAS- } \\
\text { EPAP } \\
n=314(\%)\end{array}$ & $\begin{array}{l}p \\
\text { Value }\end{array}$ \\
\hline Median gestation (IQR) & $8(6-11)$ & $8(6-10)$ & 0.304 \\
\hline Observations performed at triage (yes) & $198(74.2)$ & $236(75.2)$ & 0.782 \\
\hline Pain assessment documented (yes) & $34(12.7)$ & $112(35.7)$ & $<0.001$ \\
\hline Follow-up & & & $<0.001$ \\
\hline No follow-up & $14(5.2)$ & $8(2.5)$ & \\
\hline EPAS/ED follow-up & $84(31.5)$ & $37(11.8)$ & \\
\hline Planned repeat investigation(s) & $21(7.9)$ & $98(31.2)$ & \\
\hline O\&G follow-up & $64(24.0)$ & $92(29.3)$ & \\
\hline GP follow-up & $84(31.5)$ & $79(25.2)$ & \\
\hline Management plan & & & 0.152 \\
\hline Expectant & $202(75.7)$ & $253(80.6)$ & \\
\hline Surgical & $65(24.3)$ & $61(19.4)$ & \\
\hline
\end{tabular}

$E D$, emergency department; EPAP, early pregnancy assessment protocol; EPAS, early pregnancy assessment service; GP, general practitioner; $0 \& G$, obstetrics and gynaecology.

133000 to 183000 people) between 2006 and 2026. ${ }^{11}$ This growth in population will undoubtedly result in increased demand for government and private infrastructure and services, including emergency healthcare. ${ }^{11}$ Our study identified that 584 women presented to the ED with complications associated with early pregnancy during 2011. If trends follow those of a nearby $\mathrm{ED},{ }^{7}$ the ED where our research was conducted might expect the numbers of women presenting with early pregnancy problems to double by 2015 .

\section{Patient and service delivery outcomes}

Given pressing key performance indicators such as National Emergency Access Target being placed on hospitals within Australia to improve access, innovative ways to improve timeliness of care, without compromising patient safety are required. The EPAS-EPAP is one model of care EDs can implement in order to improve several outcomes for a particular subset of women who present to the ED as has been found in our study and elsewhere. ${ }^{4} 7$ In our study, important statistical improvements were seen in time to see a clinician (decreased by $6 \mathrm{~min}$ from 35 to $29 \mathrm{~min}$ ) and admission rate (decreased by $6 \%$ from $14.5 \%$ to $8.5 \%$ ). Although re-presentation rates to the ED increased, this is likely reflective of the change in care delivery processes; that is, scheduled ED re-presentations with resultant decreased admission rate. ED LOS was noted to decrease by $21 \mathrm{~min}$ (from 173 to $152 \mathrm{~min}$ ); however, the absence of statistical significance indicates that this difference could be due to chance. Compared with other similar evaluations, the study site performed well on most of these parameters. Wendt et $a l^{7}$ indicated that in the first year following the introduction of an EPAC model time to see a clinician decreased by $25 \mathrm{~min}$ (from 76 to $51 \mathrm{~min}$ ), ED LOS decreased by $14 \mathrm{~min}$ (290 to $276 \mathrm{~min}$ ) and admission rate decreased by $1.6 \%$ (from $31.9 \%$ to $30.3 \%$ ). Most notably, in the ensuing 4 years, further improvements continued. $^{7}$

Improvements in outcomes identified in this study were coupled with improvements in care delivery such as pathology ordering, ultrasound ordering and documentation of pain assessment. Previous research on a similar group of women at a nearby ED has reported that within its first year of EPAC operation, nursing observations were performed $80-90 \%$ of the time and pain scores were documented $55-60 \%$ of the time. ${ }^{7}$ With only around one-third of women having pain scores documented, and $75 \%$ having observations performed at triage, further room for improvement exists in these nursing care duties at our study site ED. These nursing duties are important to undertake in this group of women as they can indicate the need for more emergent care within the ED as well as enhance the decision making regarding appropriateness of EPAS referral.

\section{Models of care}

The model of care implemented in the study site primarily involved an EPAS approach with the NP on duty (in a rotating type roster) managing the care delivery process for women who presented to the ED with complications associated with early pregnancy (mainly during the hours of 07:00-23:00). At times when the NP was not on duty and after-hours, an EPAP approach was to be followed. Other models noted within the literature describe the use of a few specifically assigned and selected ED-based nurses to manage care of this patient group. Benefits of both ED trained and midwifery trained nurses have been reported. ${ }^{78}$ While we had demonstrable improvements in our study, others have reported outcomes (eg, time to see a clinician, ED LOS, reduced pathology ordering and decreased need for hospital admission) more positive than ours. ${ }^{467}$ The adjustment of the EPAS-EPAP model described in our study to one that is led by a dedicated NP, advanced practice nurse or midwife with multidisciplinary input as required may be worthwhile for further improvements in outcomes. Within Queensland, statewide guidelines to manage this group of women who present to the ED were introduced in late 2011. ${ }^{15}$ Larger scale outcomes studies are required to understand whether these guidelines are being followed and to inform where further education or care delivery improvements can be made.

Early pregnancy services alternate to the ED-based EPASEPAP model described herewithin have been noted within the literature. These include models that function in a ward/clinic setting outside of the ED but within the hospital. ${ }^{3} 416$ These models can vary in hours of operation (ie, three mornings/week or business hours only) and appear to be suited to stable patients with pain and/or bleeding who may or may not have pathology results indicating early pregnancy problems. These models (like the ED-based model) are efficient and comprehensive and can be useful in reducing ED LOS, the need for ED re-presentation and hospital admission. ${ }^{416}$ Specific benefits of the ED-based model include timeliness of service with 24/7 operational capacity, emergency clinical skill set to recognise and act on the deteriorating patient, ready access to extended services such as radiology and pathology and availability for follow-up capacity. The decision as to which model is most appropriate to implement requires careful consideration that takes account of several factors, including the servicing population and location, the political landscape within the hospital and the availability of medical and ancillary services and skills. Furthermore, a comprehensive cost comparison of an ED-based model versus non-ED-based model would be warranted.

\section{Limitations}

There were several limitations of this study. First, our sample may not reflect other regions in Australia in terms of socioeconomic status, ethnicity, departmental busyness or patient acuity (factors that may affect individual decision-making processes). Second, the time frames used (6 months pre-EPAS-EPAP 
implementation and post-EPAS-EPAP implementation) may not have been long enough to show sustained statistically significant improvements; however, there were some promising initial clinical and statistical improvements following EPAS-EPAP implementation. The time frame used for the evaluation was chosen in order to trial the model, best use funding received, collect a depth of data (ie, electronic plus chart review information for a specific time frame) and disseminate findings in a timely manner. Third, the use of retrospective data did not allow for easy identification of service received (EPAS or usual care) hampering further in-depth analysis. We were, however, able to identify clinician type (NP or MO) to inform discussion regarding practitioner management. Fourth, a cost-effectiveness analysis was beyond the scope of this study. Thus, we are limited in our ability to determine whether the ED-based EPAS-EPAP model we describe was more or less cost-effective than (i) the PRE EPAS-EPAP model where admission rates were higher, ED re-presentation rates lower and time to see a clinician longer; (ii) an ED-based EPAS (NP-led service) only model; (iii) an ED-based EPAP only model or (iv) a non-ED-based EPAS model.

\section{CONCLUSION}

Some service and clinical management improvements were identified following the implementation of the EPAS-EPAP model. Specifically, allocated/dedicated staff and resources to deliver the EPAS may result in further service improvements and contribute to a patient-centred model of care.

\footnotetext{
Author affiliations

'Department of Emergency Medicine, Redland Hospital, Cleveland, Queensland, Australia

${ }^{2}$ Capricorn Coast Hospital and Health Services, Yeppoon, Queensland, Australia

${ }^{3}$ State Wide and CQ NP Network, Queensland, Australia

${ }^{4}$ Department of Emergency Medicine, Gold Coast Hospital and Health Service, Gold Coast, Queensland, Australia

${ }^{5}$ Clinical Access and Redesign Unit, Brisbane, Queensland, Australia

${ }^{6}$ Centre for Health Practice Innovation, Griffith University, Gold Coast, Queensland, Australia

${ }^{7}$ School of Medicine, University of Queensland, Brisbane, Queensland, Australia
}

Acknowledgements We acknowledge and thank the patients and staff who participated in this study as well as funding received from the Office of the Chief Nursing Officer (OCNO).

Contributors KW, JC, CM and KB: study design; KW, JC and RS: data collection and analysis; KW, JC, CM, KB and RS: manuscript preparation.
Funding Funding was received from the Office of the Chief Nursing Officer (OCNO) to undertake this study.

Competing interests Some of the authors ( $K W, C M, K B$ and $R S$ ) were employees of the institution where this research was carried out.

Ethics approval Metro South human research ethics committee.

Provenance and peer review Not commissioned; externally peer reviewed.

Open Access This is an Open Access article distributed in accordance with the Creative Commons Attribution Non Commercial (CC BY-NC 3.0) license, which permits others to distribute, remix, adapt, build upon this work non-commercially, and license their derivative works on different terms, provided the original work is properly cited and the use is non-commercial. See: http://creativecommons.org/ licenses/by-nc/3.0/

\section{REFERENCES}

1 Everett C. Incidence and outcome of bleeding before the 20th week of pregnancy: prospective study from general practice. BMJ 1997;315:32.

2 Bacidore V, Warren N, Chaput C, et al. A collaborative framework for managing pregnancy loss in the Emergency Department. J Obstet Gynecol Neonatal Nurs 2009;38:730-8

3 Bigrigg $M$, Read $M$. Management of women referred to early pregnancy assessment unit: care and cost effectiveness. BMJ 1991;302:577-9.

4 Brownlea S, Holdgate A, Thou S, et al. Impact of an early pregnancy problem service on patient care and Emergency Department presentations. Aust N Z J Obstet Gynaecol 2005;45:108-11.

5 Swanson KM. Effects of caring, measurement, and time on miscarriage impact and women's well-being. Nurs Res 1999;48:288-98.

6 O'Rourke D, Wood S. The early pregnancy assessment project: the effect of cooperative care in the emergency department for management of early pregnancy complications. Aust N Z J Obstet Gynaecol 2009;49:110-14.

7 Wendt K, Crilly J, Beatson N. An evaluation of early pregnancy outcomes in one Australian Emergency Department: part 2. Australas Emerg Nurs J 2012:15:77-85.

8 Wattimena J, Pelosi M, Cheney $\mathrm{K}$, et al. Factors affecting length of stay for women presenting with early pregnancy complications to a public hospital emergency department. Emerg Med Australas 2013;25:22-7.

9 Crilly J, Wendt K, Beatson N. A structure and process evaluation of an Early Pregnancy Assessment Clinic (EPAC) in one Australian Emergency Department: part 1. Australas Emerg Nurs J 2012;15:68-76.

10 Redland City Council. Demographic Profile. 2011. http://www.redland.qld.gov.au/ AboutRedlands/DemographicProfile/Pages/default.aspx (accessed 27 Mar 2013).

11 Queensland Health. Redland Hospital Overview. 2011. http://qheps.health.qld.gov. au/bay/pdf/overview_redland.pdf (accessed 27 Mar 2013).

12 World Health Organisation. International Classification of Diseases. 2007. http:// www.who.int/classifications/icd/en/ (accessed 9 May 2013).

13 Snell BJ. Assessment and management of bleeding in the first trimester of pregnancy. J Midwifery Wom Heal 2009;54:483-91.

14 Australasian College for Emergency Medicine. The Australasian triage scale. Emerg Med 2002:14:335-6.

15 Queensland Maternity and Neonatal Clinical Guidelines Program. Early pregnancy loss. 2011. http://www.health.qld.gov.au/qcg/ (accessed 27 Mar 2013).

16 Tunde-Byass $M$, Cheung V. The value of the early pregnancy assessment clinic in the management of early pregnancy complications. J Obstet Gynaecol Can 2009;31:841-4. 